Reor i ent at i onal rel axat i on of ar omat i c mol ecul es in the mol ecul ar cavity of crystal I i ne syndi ot act i c pol ystyrene st udi ed by nol ecul ar dynamics si mul at i on

\begin{tabular}{|l|l|}
\hline 著者 & $\begin{array}{l}\text { TAMAl Yoshi nor i , TSUJ TA Yoshi har u, FUKUDA } \\
\text { M t suhi ro }\end{array}$ \\
\hline $\begin{array}{l}\text { j our nal or } \\
\text { publ i cat i on ti t l e }\end{array}$ & Journal of Nol ecul ar Struct ure \\
\hline vol une & 739 \\
\hline number & 1 \\
\hline page r ange & $33-40$ \\
\hline year & $2005-04$ \\
\hline URL & ht t p: //hdl . handl e. net /10098/1165 \\
\hline
\end{tabular}




\title{
Reorientational relaxation of aromatic molecules in the molecular cavity of crystalline syndiotactic polystyrene studied by molecular dynamics simulation
}

\author{
Yoshinori Tamai $^{\mathrm{a}, *}$, Yoshiharu Tsujita ${ }^{\mathrm{b}}$, Mitsuhiro Fukuda $^{\mathrm{c}}$

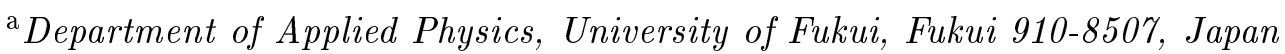 \\ ${ }^{\mathrm{b}}$ Department of Materials Science and Engineering, Nagoya Institute of \\ Technology, Showa-ku, Nagoya 466-8555, Japan \\ ${ }^{\mathrm{c}}$ Textile Materials Science Laboratory, Hyogo University of Teacher Education, \\ Yashiro-cho, Hyogo 673-1494, Japan
}

\begin{abstract}
The reorientational motion of aromatic molecules, which are clathrated in the molecular cavity of the crystalline syndiotactic polystyrene $\delta$ form was investigated using a molecular dynamics simulation. The in-plane and out-of-plane reorientation of phenyl rings and the rotational motion of methyl groups were examined for the guest species: benzene, toluene, $p$-xylene, $m$-xylene, $o$-xylene, and mesitylene. The cavity structures, the host-guest interactions, and the resultant effects on the reorientational dynamics of the guests were investigated in detail. The reorientational relaxation time obeyed the Arrhenius law over a wide temperature range. The relaxation time was found to be significantly affected by the difference in the molecular structure of the guests, as a consequence of the variety in the host-guest interaction.
\end{abstract}

Key words: polystyrene, clathrate, reorientational relaxation, simulation 


\section{Introduction}

Benzene molecules in the pure crystal can undergo reorientational jumping motions about its $\mathrm{C}_{6}$ symmetry axis [1]. This kind of motion is also observed in the clathrate organic or organometallic complexes [2,3], which encage aromatic molecules. The reorientational dynamics of the aromatic molecules in the clathrate compounds is of much interest because it reflects the guest-host interaction and the cavity structure of the host matrix.

Syndiotactic polystyrene (s-PS) forms a clathrate molecular compound, which are called the $\delta$ form, with various solvents, e.g., toluene [4,5], iodine [6], and 1,2-dichloroethane [7]. The emptied $\delta_{e}$ form [8] can be obtained by the extraction of the solvent molecules from the $\delta$ form. In the $\delta$ form, the solvent molecules are clathrated in the individual molecular size cavities. The solidstate ${ }^{2} \mathrm{H}$ NMR spectra [9] suggested the fast reorientation of benzene guests in the $\delta$ form, though no quantitative information about the relaxation time and the activation energy has been reported. The theoretical and simulation studies on the reorientational dynamics of benzene in a neat liquid and solutions have also been reported [10-12]. It is expected that the character of the dynamics is quite different between that in the clathrate forms and that in the random structures.

In this study, a molecular dynamics (MD) simulation was performed for various $\delta$ form crystals in order to investigate the reorientational dynamics of the aromatic guests. The reorientational dynamics of benzene, toluene, and $p$-xylene, which are clathrated in the $\delta$ form, has been partially reported in a

* Corresponding author. Tel.:+81-776-27-8032; Fax: +81-776-27-8032 Email address: tamai@polymer.apphy.fukui-u.ac.jp (Yoshinori Tamai). 
preliminary study [13]. In addition to these three guest species, the $\delta$ forms which encage $m$-xylene, $o$-xylene, and mesitylene have also been simulated in this study. The reorientational motion of the aromatic rings and the rotational motion of the substituent methyl groups are analyzed in terms of the cavity structures. The host-guest interactions and the resultant effects on the reorientational dynamics of the guests are investigated in detail.

\section{Simulation details}

The potential model and the procedure for the MD simulation are the same as those used in the the previous studies [13-17]. The potential parameters were taken from the all-atom force field, AMBER [18]. The weak partial charges were assigned only to the aromatic hydrogen $(+0.085 e)$ and aromatic carbon

which is bonded to the hydrogen $(-0.085 e)$ [19]. The long-range nonbonded interactions were smoothly cut off at $14 \AA$. The initial structures of the $\delta$ forms were generated on the basis of an X-ray diffraction experiment on the s-PS/toluene $\delta$ form [5]. The toluene molecules were replaced by each aromatic solvent. An MD unit cell contains $3 \times 4 \times 6$ crystal units; the number of guest molecules is 144 . The total numbers of atoms are 10944-12240, depending on the guests. The three-dimensional periodic boundary condition was applied. The crystal was modeled as a single crystal in which the monomer units are infinitely connected along the $c$-axis.

First, the MD simulations were performed for 100 ps for each crystal at $300 \mathrm{~K}$ under ambient pressure, after an equilibration run of 50 ps. The temperature was then increased or decreased in $50 \mathrm{~K}$ interval steps in the range of 200$800 \mathrm{~K}$. An equilibration run of 20 ps, followed by a sampling run of 100 ps, 
was performed at each temperature. Long time runs up to 2000 ps were also performed at each temperature in order to obtain the long-time correlation functions. Temperature and pressure tensors were controlled by the Nosé [20] and Parrinello-Rahman [21] methods, respectively. The equations of motion were solved using a variant of the Verlet algorithm $[22,23]$ with a time step of 1 fs. The bond lengths were constrained by the SHAKE method [24].

The MD simulations were also performed for pure solid benzene containing $5 \times 5 \times 5$ crystal units (500 benzene molecules). The crystal system of benzene is orthorhombic and the space group is Pbca. Four molecules are confined in a crystal unit cell [25]. The partial charge values of $\pm 0.146 e$ were used for the simulation of pure benzene [26]. For the simulation of pure benzene, the temperature was increased or decreased in $25 \mathrm{~K}$ interval steps from the initial temperature of $200 \mathrm{~K}$ in the range of $100-425 \mathrm{~K}$.

The trajectories of the atoms were recorded every 500 steps (500 fs) for later analysis. Furthermore, in order to measure rapid-motion time correlation functions, short-time MD simulations of 10 and $50 \mathrm{ps,} \mathrm{in} \mathrm{which} \mathrm{the} \mathrm{trajectories}$ were recorded every 5 and 50 steps, respectively, were also performed at each temperature, using the last coordinates of the long-time runs as initial structures.

A cluster machine with 28 processors (Intel Xeon and Pentium 4) was used for the simulation along with the molecular simulation program PAMPS $[27,28]$ coded by one of the authors (Y.T.). 


\section{Results and discussion}

\subsection{Stability of Crystal}

The snapshot of the s-PS/benzene $\delta$ form is shown in Fig. 1. The aromatic molecules are clathrated in the molecular cavities of the s-PS crystal. The aromatic ring planes are nearly parallel to the $a-b$ plane. The densities and crystal cell dimensions of the $\delta$ forms are listed in Table 1, compared with the experiments. Those of solid benzene are also listed in Table 2. The crystal structures were stable during the simulation runs. The experimental density and cell dimensions were well reproduced by the simulation. The length of the $b$-axis of the $\delta$ forms was extended by increasing the steric hindrance, associated with the opening of the angle $\gamma$.

The temperature dependence of the density of each crystal is shown in Fig. 2. The crystal of solid benzene was molten at $375 \mathrm{~K}$ by way of the first order phase transition. The melting temperature is higher than the experimental value, $278.683 \mathrm{~K}$. A kind of second order phase transition was also observed at $300 \mathrm{~K}$, where the symmetry was changed from orthorhombic to cubic. We do not consider this phase transition behavior anymore because it is outside the aim of this paper. Only the trajectories below $275 \mathrm{~K}$ were used for the analysis of solid benzene. The $\delta$ forms were molten at around $700 \mathrm{~K}$, which is higher than the melting point of s-PS, $550 \mathrm{~K}$. The superheated state could easily be attained in the simulation because of the restriction of the unit cell and the infinite connectivity of the main chains along the $c$-axis. The orientation and reorientational dynamics of the guests in the solid state are presented below. 


\subsection{Orientation of guest molecules}

In order to measure the orientation of the guests, we defined two types of unit vectors embedded on the phenyl rings: (1) the unit vector along the chord from the $\mathrm{C}_{1}$ to $\mathrm{C}_{4}$ carbons, $\mathbf{u}_{\|}$, and (2) the unit vector perpendicular to the ring plane, $\mathbf{u}_{\perp}$. The former probes the reorientation about the $C_{6}$ symmetry axis of the phenyl rings (for benzene), while the latter probes the flip motion of the ring planes. The time evolution of the orientational angles of $\mathbf{u}_{\|}$and $\mathbf{u}_{\perp}, \phi$ and $\theta$, respectively, were calculated from the trajectories. The former is the angle of the projection vector of $\mathbf{u}_{\|}$on the $a-b$ plane measured from the $a$-axis, and the latter is the angle of the projection of $\mathbf{u}_{\perp}$ on the $c$ - $a$ plane measured from the $c$-axis. In our simulation system, the $a$-axis just coincides with the $x$-axis of the global coordinate system; the $c$-axis slightly fluctuates around the $z$-axis.

In order to probe the rotational motion of the methyl groups, which are attached to the phenyl rings of the guests, the motion of the unit vector normal to the $\mathrm{C}-\mathrm{C}-\mathrm{H}$ plane of the $\mathrm{C}-\mathrm{CH}_{3}$ group, $\mathbf{u}_{\mathrm{Me}}$, was also monitored. The reorientational motion of the methyl groups about the $C_{3}$ symmetry axis can be probed by this vector.

The time evolution of the angles $\phi$ and $\theta$ of the benzene at $250 \mathrm{~K}$ and toluene at $400 \mathrm{~K}$ in the $\delta$ form is shown in Fig. 3. No flip motion was observed at this temperature, by considering $\theta$. The reorientational jumping motion of $60^{\circ} \mathrm{cy}-$ cles about $C_{6}$ symmetry axis is clearly confirmed from the angle $\phi$ of benzene. This kind of motion is experimentally observed in pure crystalline benzene [1] and many benzene clathrates $[2,3]$. The fast reorientation of benzene in the $\delta$ 
form was also observed in the ${ }^{2} \mathrm{H}$ NMR experiment of Trezza et al. [9]. The in-plane reorientation of toluene was also observed in the simulation at $400 \mathrm{~K}$. The interval angle of the jumps was estimated from Fig. 3(a) as $180^{\circ}$, which is different from that of benzene, $60^{\circ}$. The symmetry of the cavity and the guests was reflected by their angle.

The distributions of the orientational angle $\phi$ at $300 \mathrm{~K}$ are shown in Fig. 4(a). Note that half of the guest molecules was $\phi=38^{\circ}$ and the others were $\phi=-142^{\circ}$ for the initial crystal structures. The distribution for benzene reflects the reorientational jumping motion of $60^{\circ}$ cycles. On the other hand, no jumping reorientation was observed for $p$-xylene. Only a few jumps in $\phi$ every $180^{\circ}$ were observed for toluene. The distribution of $\phi$ for toluene and $o-$ xylene is rather broad as a consequence of a large amplitude swinging motion [2]. The most probable angles for $\phi$ are different for the different guest species: $62^{\circ}, 53^{\circ}, 38^{\circ}, 5^{\circ},-59^{\circ}$, and $80^{\circ}$ for benzene, toluene, $p$-xylene, $m$-xylene, $o$ xylene, and mesitylene, respectively. The distributions of angle $\theta$ are shown in Fig. 4(b). Only the fluctuation around $0^{\circ}$ was observed for $\theta$ at $300 \mathrm{~K}$. The half width at half maximum (HWHM) of $\theta$ at $300 \mathrm{~K}$ was $13^{\circ}$, which corresponds to the fluctuation of the $C_{6}$ symmetry axis. The aromatic guest molecules are restricted on the plane with a slight deviation.

The snapshots of the guest molecules, which are encaged in the cavities, are shown in Fig. 5. The most probable orientations are indicated by the arrows. The shape of the cavities was derived from the cluster analysis of the accessible volumes, which were calculated for a probe of radius $1 \AA$ [16]. The colored region in the figure shows the continuously accessible regions for a probe of radius $1 \AA$. The three guest species, benzene, toluene, and $p$-xylene, fit quite naturally in the cavity, while a significant deformation of the cavity shape 
is caused by $m$-xylene, $o$-xylene, and mesitylene. Because the substitution of the $p$-position matches the symmetry of the cavity, the complex of $p$-xylene is probably more stable than that of $m$-xylene. This agrees with the experimental results that $p$-xylene is preferentially absorbed by the $\delta$ form from a mixed solvent of $m$ - and $p$-xylene [29].

\subsection{Relaxation time}

The rotational motion of the aromatic molecules was quantitatively analyzed using the reorientational time correlation function:

$$
C_{l}(t)=\left\langle P_{l}[\mathbf{u}(0) \cdot \mathbf{u}(t)]\right\rangle
$$

where $P_{l}(x)$ is the $l$ th order Legendre polynomial, $\mathbf{u}(t)$ is a unit vector embedded in the molecule at time $t$, and $\langle\ldots\rangle$ denotes the ensemble average. The vectors, $\mathbf{u}_{\|}, \mathbf{u}_{\perp}$, and $\mathbf{u}_{\mathrm{Me}}$, which are defined above, were used as $\mathbf{u}(t)$. The decay curves for $l=1$ and $l=2$, i.e.,

$$
C_{1}(t)=\langle\mathbf{u}(0) \cdot \mathbf{u}(t)\rangle
$$

and

$$
C_{2}(t)=\frac{1}{2}\left\langle 3[\mathbf{u}(0) \cdot \mathbf{u}(t)]^{2}-1\right\rangle
$$

were examined in this study.

Fig. 6 shows the decay curves of $C_{1}(t)$ and $C_{2}(t)$, which were calculated from $\mathbf{u}_{\|}(t)$ at $300 \mathrm{~K}$ for the in-plane reorientation of the phenyl rings. After a rapid decay shorter than $1 \mathrm{ps}$, the $C_{l}(t)$ curve decays during a characteristic time scale for each guest species. That of benzene relaxes faster than those of the other guests, which have the methyl side groups. As for benzene, the 
values of $C_{1}(t)$ converge on zero during 80 ps while those of $C_{2}(t)$ converge on the non-zero value, 0.22 . If the rotational motion is constrained on a ring plane, the $C_{2}(t)$ curve converges on 0.25 and 1 for the reorientation of $60^{\circ}$ and $180^{\circ}$ cycles, respectively. Therefore, the guest molecules are approximately constrained on the ring planes with slight deviations, as can also be confirmed from the distribution of angle $\theta$ (Fig. 4).

Fig. 7 shows the decay curves for the reorientation of the methyl groups, which are calculated from $\mathbf{u}_{\mathrm{Me}}(t)$ at $300 \mathrm{~K}$. The relaxation is more than two order faster than that of the phenyl ring reorientation. If the methyl groups strongly interact with the "wall" of the cavities, the reorientational motion of the methyl groups is strongly hindered. The strong interaction is implied from the significant deformation of the cavity shape for the guest species: $m$-xylene, $o$-xylene, and mesitylene (see Fig. 5). Therefore, the reorientational motion of the methyl groups of those guests is slower than that of $p$-xylene and toluene, for which the cavity shape is not deformed. The remarkably slower relaxation for $o$-xylene is the consequence of a significant deformation of the cavity shape and also of the strong interaction between two methyl groups at the $o$-position.

As shown in Fig. 8, the curves of $C_{1}(t)$ can be satisfactorily fitted to the sum of the Gaussian and exponential functions:

$$
C_{1}(t)=(1-A) \exp \left[-\left(t / \tau_{s}\right)^{2}\right]+A \exp (-t / \tau)
$$

where $A, \tau_{s}$, and $\tau$ are fitting parameters. The short relaxation times, $\tau_{s} \sim 0.5$ ps, were almost constant for all the temperatures, while the main (long) relaxation times, $\tau$, increased on decreasing the temperature. The temperature dependence of $\tau$ for the phenyl ring and methyl group reorientation is shown in Fig. 9 and 10, respectively. The reorientational relaxation times obeyed the 
Arrhenius law over a wide temperature range. The calculated value of $\tau$ in solid benzene at $200 \mathrm{~K}$ is in good agreement with the experimental value of $1.41 \times 10^{-9} \mathrm{~s}[1]$. The simulation values in the $\delta$ form cannot be directly compared with the experiments because the relaxation time was not determined in the experiment [9]. The relative relaxation times of the methyl groups for the different guest species may be confirmed by the NMR chemical shift, because it reflects the interaction between the guests and the cavity wall. A quantitative experimental study is desired.

The $\tau$ values are well fitted by the equation:

$$
\tau=\tau_{0} \exp \left(E_{A} / R T\right)
$$

where $R$ is the gas constant and $E_{A}$ is the activation energy. Because the relaxation time obeys the Arrhenius law, the relaxation process is governed by a single activation process over a wide temperature range. The $E_{A}$ values are listed in Table 3. The activation energy of the in-plane reorientation of benzene in the $\delta$ form, $12.8 \mathrm{~kJ} / \mathrm{mol}$, is lower than that in the solid benzene, $18.4 \mathrm{~kJ} / \mathrm{mol}$. The relaxation time of benzene in the $\delta$ form is shorter than that in the solid benzene. The benzene molecule in the $\delta$ form rotates more easily than in the pure solid. The benzene molecule in the $\delta$ form is a good molecular rotator. As shown in Fig. 9(b), the $180^{\circ}$ flip motion of $\mathbf{u}_{\perp}$ (the out-ofplane motion of rings) was also observed above $500 \mathrm{~K}$. The activation energy, $54.9 \mathrm{~kJ} / \mathrm{mol}$, is quite high because the out-of-plane reorientation requires a significant deformation of the cavity shape. In liquid benzene, the activation energies were determined by the MD simulation to be 7.1 and $9.2 \mathrm{~kJ} / \mathrm{mol}$ for the in-plane and out-of-plane reorientational motions, respectively [11]. The large difference in $E_{A}$ between the in-plane $(12.8 \mathrm{~kJ} / \mathrm{mol})$ and out-of-plane 
$(54.9 \mathrm{~kJ} / \mathrm{mol})$ motion is a characteristic of the reorientation in the $\delta$ form.

The activation energy of the in-plane motion of $p$-xylene is quite high and that of toluene is intermediate between benzene and $p$-xylene. As for $p$-xylene, the in-plane reorientation is harder than that out-of-plane because the two methyl groups in the $p$-position act as a kind of anchor bolt. The $E_{A}$ values of the methyl group rotation, which are also listed in Table 3, reflect the interaction between the guests and the cavity "wall". Those for toluene and $p$-xylene are smaller than those of $m$-xylene, $o$-xylene, and mesitylene.

In the laboratory experiments, the $\delta$ form is transformed into the $\gamma$ form above $400 \mathrm{~K}$; the aromatic guest molecules are released from the cavities. In the present simulation, the crystal structures were held up to $650 \mathrm{~K}$. Simulations under the experimentally inaccessible condition are also meaningful to understand the mechanism of dynamics. The extrapolation of $\tau$ in Fig. 9(b) implies that the out-of-plane motion may be observed at lower temperature on the experimental time scale, though it was only observed at high temperature in the simulation, in which the observation time is $\sim 10^{-9} \mathrm{~s}$. Because the individual cavities are separated from each other by the polymer matrix in the $\delta$ form $[16,30,31]$, the lattice spacing should be significantly extended in order for large molecules to diffuse into the crystal. The guest desorption mechanism is still unclear. The out-of-plane motion of the guests may have a close relation to the desorption mechanism. 


\section{Conclusions}

The reorientational relaxation time of the aromatic molecules, which are clathrated in the molecular cavity, is significantly affected by the molecular structure of the guests. This is a consequence of the variety in the host-guest interactions. The cavity shape is deformed by the clathration of guests, and the molecular motion of the guests is hindered by the interaction between the guests and the cavity wall. The investigation of the reorientational dynamics of the guests in the clathrate compounds is essential for understanding the guest-host interaction and the cavity structure of the host matrix.

\section{Acknowledgments}

This research was supported by the Ministry of Education, Science, Sports and Culture, Grant-in-Aid for Scientific Research on Priority Area, 13133203, and Grant-in-Aid for Young Scientists (B), 15750100.

\section{References}

[1] E.R. Andrew, R.G. Eades, Proc. R. Soc. Lond. A 218 (1953) 537.

[2] D. Braga, Chem. Rev. 92 (1992) 633.

[3] S. Nishikiori, T. Kitazawa, C. H. Kim, T. Iwamoto, J. Phys. Chem. A 104 (2000) 2591.

[4] Y. Chatani, Y. Shimane, Y. Inoue, T. Inagaki, T. Ishioka, T. Ijitsu, T. Yukinari, Polymer, 33 (1992) 488. 
[5] Y. Chatani, Y. Shimane, T. Inagaki, T. Ijitsu, T. Yukinari, H. Shikuma, Polymer, 34 (1993) 1620.

[6] Y. Chatani, T. Inagaki, Y. Shimane, H. Shikuma, Polymer, 34 (1993) 4841.

[7] C. de Rosa, P. Rizzo, O.R. de Ballesteros, V. Petraccone, G. Guerra, Polymer, 40 (1999) 2103.

[8] C. de Rosa, G. Guerra, V. Petraccone, B. Pirozzi, Macromolecules, 30 (1997) 4147.

[9] E. Trezza, A. Grassi, Macromol. Rapid Commun. 23 (2002) 260.

[10] F. Müller-Plathe, Macromolecules, 29 (1996) 4782.

[11] R. Witt, L. Sturz, A. Dölle, F. Müller-Plathe, J. Phys. Chem. A 104 (2000) 5716.

[12] R. Chelli, G. Cardini, M. Ricci, P. Bartolini, R. Righini, S. Califano, Phys. Chem. Chem. Phys. 3 (2001) 2803.

[13] Y. Tamai, M. Fukuda, Chem. Phys. Lett. 371 (2003) 620.

[14] Y. Tamai, M. Fukuda, Macromol. Rapid Commun. 23 (2002) 891.

[15] Y. Tamai, M. Fukuda, Chem. Phys. Lett. 371 (2003) 217.

[16] Y. Tamai, M. Fukuda, Polymer, 44 (2003) 3279.

[17] Y. Tamai, M. Fukuda, Trans. Mater. Res. Soc. Jpn. in press.

[18] W.D. Cornell, P. Cieplak, C.I. Bayly, I.R. Gould, K.M. Merz, Jr., D.M. Ferguson, D.C. Spellmeyer, T. Fox, J.W. Caldwell, P.A. Kollman, J. Am. Chem. Soc. 117 (1995) 5179.

[19] G.D. Smith, C. Ayyagari, R.L. Jaffe, M. Pekny, A. Bernarbo, J. Phys. Chem. A $102(1998) 4694$. 
[20] S. Nosé, J. Chem. Phys. 81 (1984) 511.

[21] M. Parrinello, A. Rahman, J. Appl. Phys. 52 (1981) 7182.

[22] L. Verlet, Phys. Rev. 159 (1967) 98.

[23] M. Ferrario, J.P. Ryckaert, Mol. Phys. 54 (1985) 587.

[24] J.P. Ryckaert, G. Ciccotti, H.J.C. Berendsen, J. Comput. Phys. 23 (1977) 327.

[25] E.G. Cox, D.W.J. Cruickshank, J.A.S. Smith, Proc. R. Soc. Lond. A 247 (1958) 1.

[26] S.T. Howard, J.A. Platts, K. Woźniak, Chem. Phys. Lett. 239 (1995) 267.

[27] Y. Tamai, H. Tanaka, K. Nakanishi, Macromolecules, 27 (1994) 4498.

[28] Y. Tamai, H. Tanaka, K. Nakanishi, Macromolecules, 28 (1995) 2544.

[29] M. Sivakumar, Y. Yamamoto, D. Amutharani, Y. Tsujita, H. Yoshimizu, T. Kinoshita, Macromol. Rapid Commun. 23 (2002) 77.

[30] G. Guerra, G. Milano, V. Venditto, P. Musto, C. de Rosa, L. Cavallo, Chem. Mater. 12 (2000) 363.

[31] G. Milano, V. Venditto, G. Guerra, L. Cavallo, P. Ciambelli, D. Sannino, Chem. Mater. 13 (2001) 1506. 
Table 1

Density and lattice constants of crystal unit cell of the s-PS $\delta$ form, compared with those of the empty $\delta_{e}$ form.

\begin{tabular}{|c|c|c|c|c|c|}
\hline guest & $\rho\left(\mathrm{g} / \mathrm{cm}^{3}\right)$ & $a(\AA)$ & $b(\AA)$ & $c(\AA)$ & $\gamma(\operatorname{deg})$ \\
\hline \multicolumn{6}{|c|}{ Simulation $(300 \mathrm{~K})$} \\
\hline empty & 0.959 & 17.38 & 11.73 & 7.81 & 115.0 \\
\hline benzene & 1.075 & 17.51 & 13.34 & 7.75 & 122.4 \\
\hline toluene & 1.091 & 17.47 & 13.42 & 7.79 & 122.0 \\
\hline$p$-xylene & 1.112 & 17.62 & 13.30 & 7.86 & 122.0 \\
\hline$m$-xylene & 1.087 & 17.74 & 14.94 & 7.77 & 123.8 \\
\hline$o$-xylene & 1.102 & 17.38 & 14.40 & 7.88 & 126.9 \\
\hline mesitylene & 1.089 & 17.91 & 14.66 & 7.85 & 127.4 \\
\hline \multicolumn{6}{|c|}{ Experiment } \\
\hline empty $^{a}$ & 0.977 & 17.4 & 11.85 & 7.70 & 117 \\
\hline toluene $^{b}$ & 1.11 & 17.58 & 13.26 & 7.71 & 121.2 \\
\hline
\end{tabular}


Table 2

Density and lattice constants of crystal unit cell of solid benzene.

\begin{tabular}{llll}
$\rho\left(\mathrm{g} / \mathrm{cm}^{3}\right)$ & $a(\AA)$ & $b(\AA)$ & $c(\AA)$ \\
\hline
\end{tabular}

Simulation $(275 \mathrm{~K})$

$\begin{array}{llll}1.020 & 7.60 & 9.32 & 7.18\end{array}$

Experiment $^{a}(270 \mathrm{~K})$

$\begin{array}{llll}1.022 & 7.46 & 9.66 & 7.03\end{array}$

${ }^{a}$ Reference [25]. 
Table 3

Activation energy of reorientational relaxation $(\mathrm{kJ} / \mathrm{mol})$.

\begin{tabular}{|c|c|c|c|}
\hline molecule & $\mathbf{u}_{\|}$ & $\mathbf{u}_{\perp}$ & $\mathbf{u}_{\mathrm{Me}}$ \\
\hline \multicolumn{4}{|c|}{$\delta$ form } \\
\hline benzene & 12.8 & 54.9 & \\
\hline toluene & 36.6 & 63.2 & 3.6 \\
\hline$p$-xylene & 98.2 & 57.1 & 3.6 \\
\hline$m$-xylene & 37.8 & 91.0 & 5.2 \\
\hline$o$-xylene & 26.6 & 107.4 & 7.7 \\
\hline mesitylene & 51.9 & 103.7 & 6.0 \\
\hline \multicolumn{4}{|c|}{ pure solid } \\
\hline benzene & 18.4 & & \\
\hline \multicolumn{4}{|c|}{ pure liquid } \\
\hline benzene $^{a}$ & 7.1 & 9.2 & \\
\hline
\end{tabular}

${ }^{a}$ Reference [11]. 
Fig. 1. Snapshot of the s-PS/benzene $\delta$ form after the sampling run of 100 ps at 300 K: (blue) s-PS, (orange) benzene. Hydrogen atoms are not shown for simplicity.

Fig. 2. Temperature dependence of the density: (a) the s-PS $\delta$ forms, and (b) solid benzene.

Fig. 3. Time evolution of the orientational angles of benzene at $250 \mathrm{~K}$ and toluene at $400 \mathrm{~K}$ in the $\delta$ form: (a) in-plane rotational angle, $\phi$, and (b) out-of-plane rotational angles, $\theta$.

Fig. 4. The distribution of the rotational angles, $\phi$ and $\theta$, of aromatic molecules in the s-PS $\delta$ form at $300 \mathrm{~K}$ : (a) in-plane, and (b) out-of-plane reorientation.

Fig. 5. Snapshots of aromatic guest molecules in the molecular cavities of s-PS $\delta$ form. Hydrogen atoms are not shown for simplicity. The cavities are projected on the $a$ - $b$ plane. The thickness along $c$-axial direction is indicated by the color scale. The most probable angles of the molecular orientation are shown by arrows.

Fig. 6. The reorientational time correlation functions calculated for the in-plane motion of the phenyl rings in the s-PS $\delta$ form at $300 \mathrm{~K}$ : (a) $C_{1}(t)$ and (b) $C_{2}(t)$

Fig. 7. The reorientational time correlation functions calculated for the methyl groups of the aromatic molecules in the s-PS $\delta$ form at $300 \mathrm{~K}$ : (a) $C_{1}(t)$ and (b) $C_{2}(t)$

Fig. 8. Fitting of the reorientational time correlation function of the in-plane motion of benzene in the $\delta$ form at $250 \mathrm{~K}$ and that of the methyl groups of $p$-xylene at 200 K. The functions are fitted to $g(t)+f(t)$, where $g(t)=(1-A) \exp \left[-\left(t / \tau_{s}\right)^{2}\right]$ and $f(t)=A \exp [-(t / \tau)]$ 
Fig. 9. Temperature dependence of (a) in-plane and (b) out-of-plane reorientational relaxation times of aromatic molecules in the $\delta$ form: (solid circle) benzene, (open square) toluene, (solid triangle) $p$-xylene, (open triangle) $m$-xylene, (solid square) $o$-xylene, and (diamond) mesitylene. That in the solid benzene is also shown (open circle).

Fig. 10. Temperature dependence of reorientational relaxation time of the methyl group about the $C_{3}$ symmetry axis: (solid circle) benzene, (open square) toluene, (solid triangle) $p$-xylene, (open triangle) $m$-xylene, (solid square) $o$-xylene, and (diamond) mesitylene in the $\delta$ form. 
Figure 1 (Y. Tamai et al.)
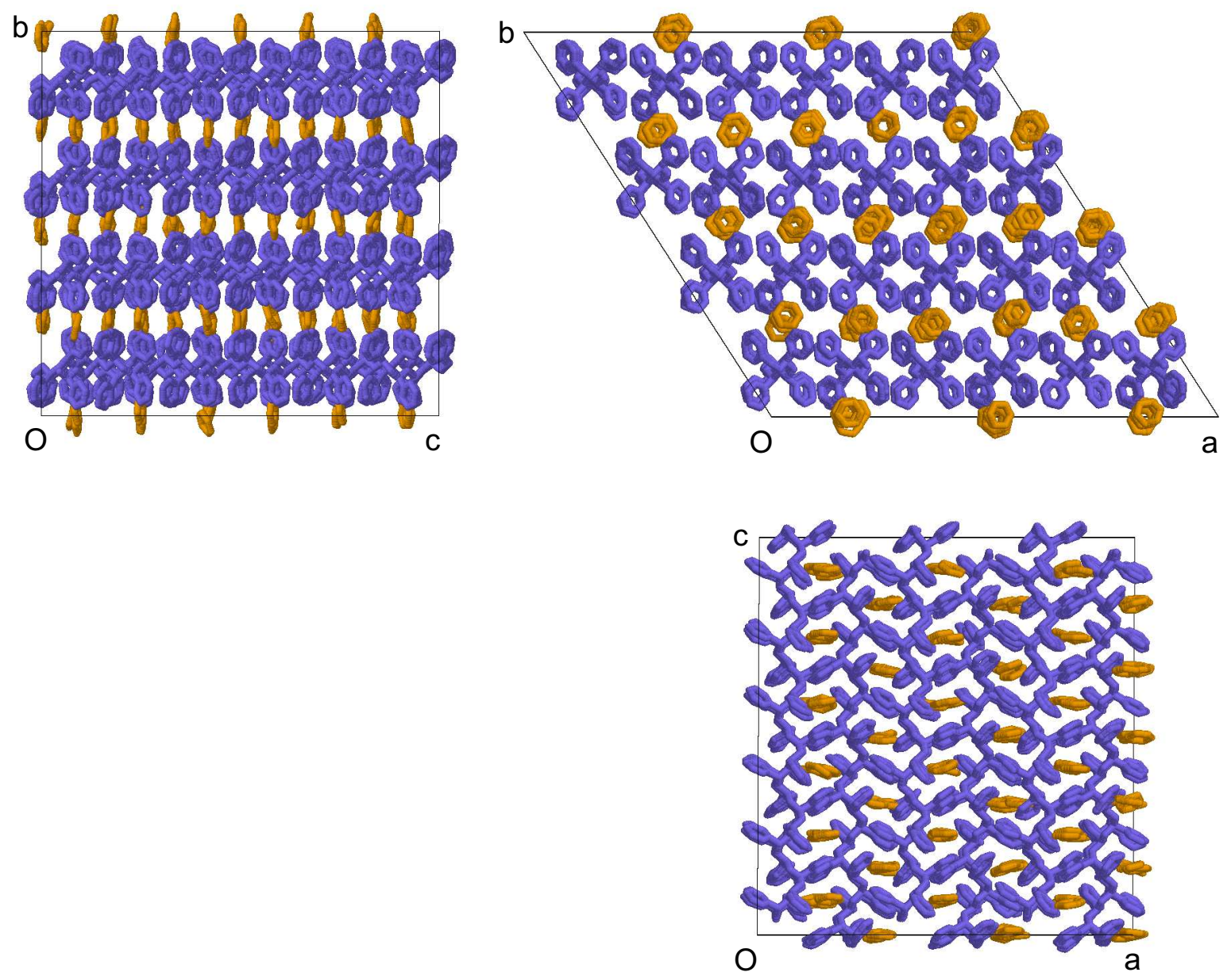
Figure 2 (Y. Tamai et al.)
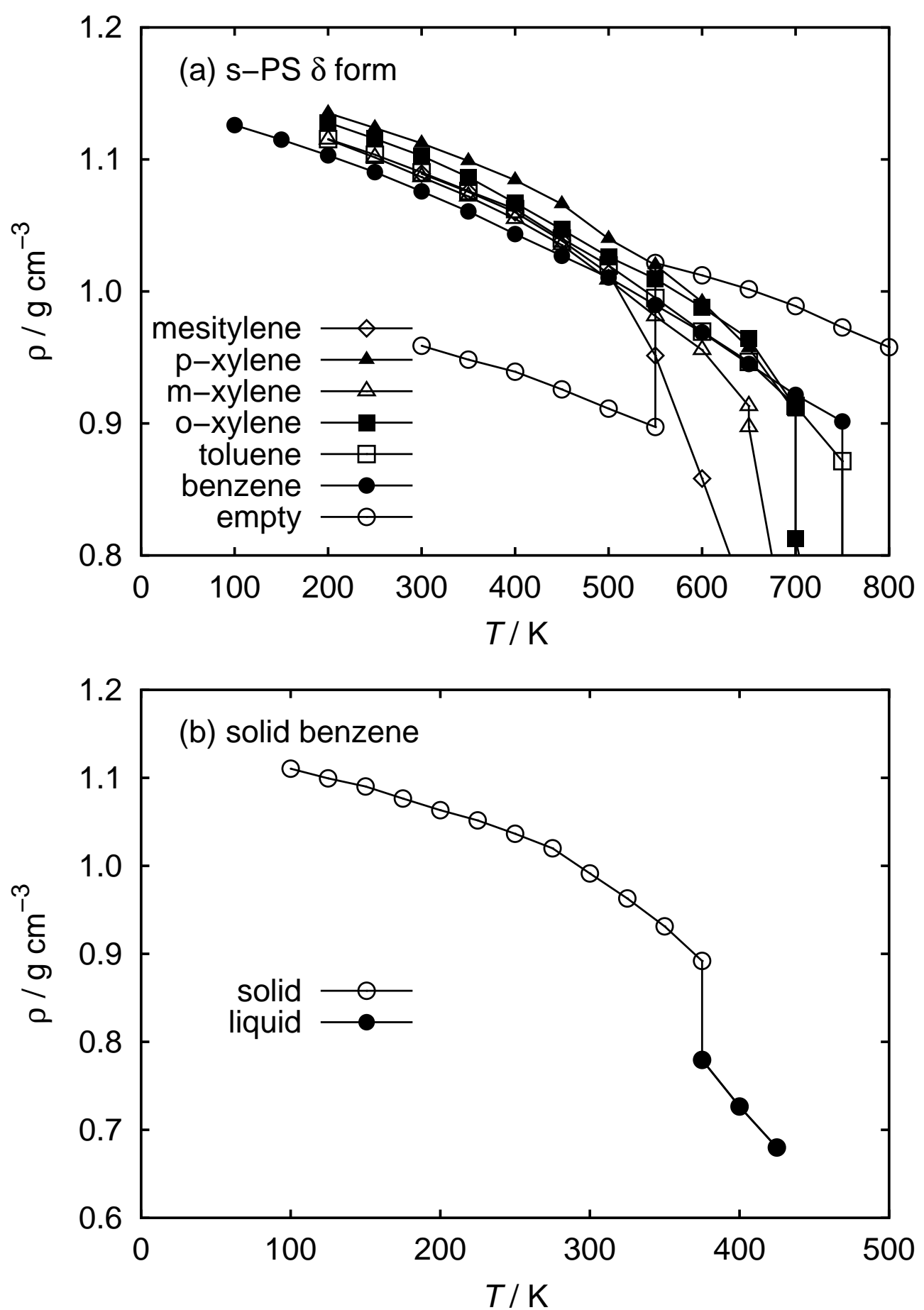
Figure 3 (Y. Tamai et al.)
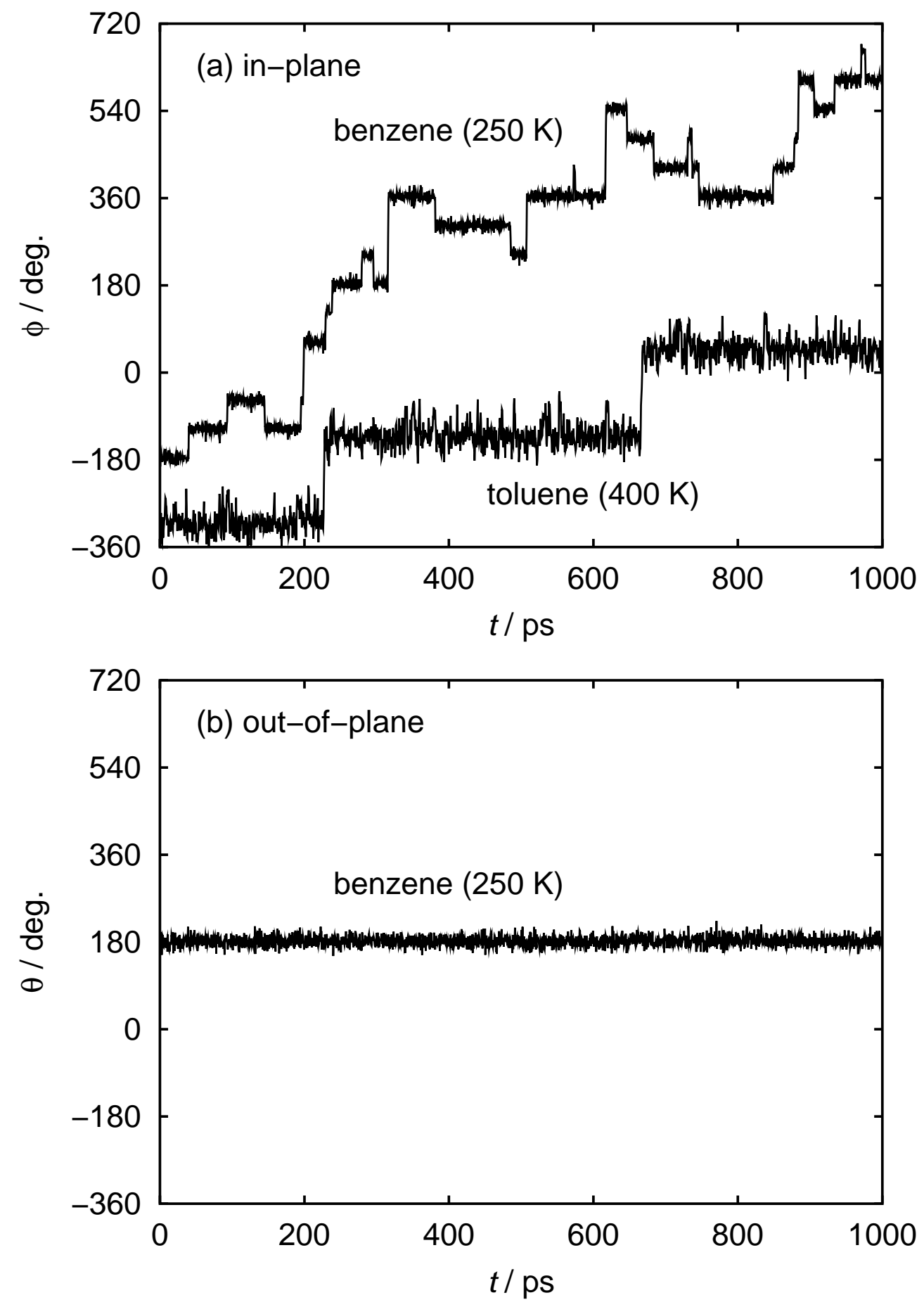
Figure 4 (Y. Tamai et al.)
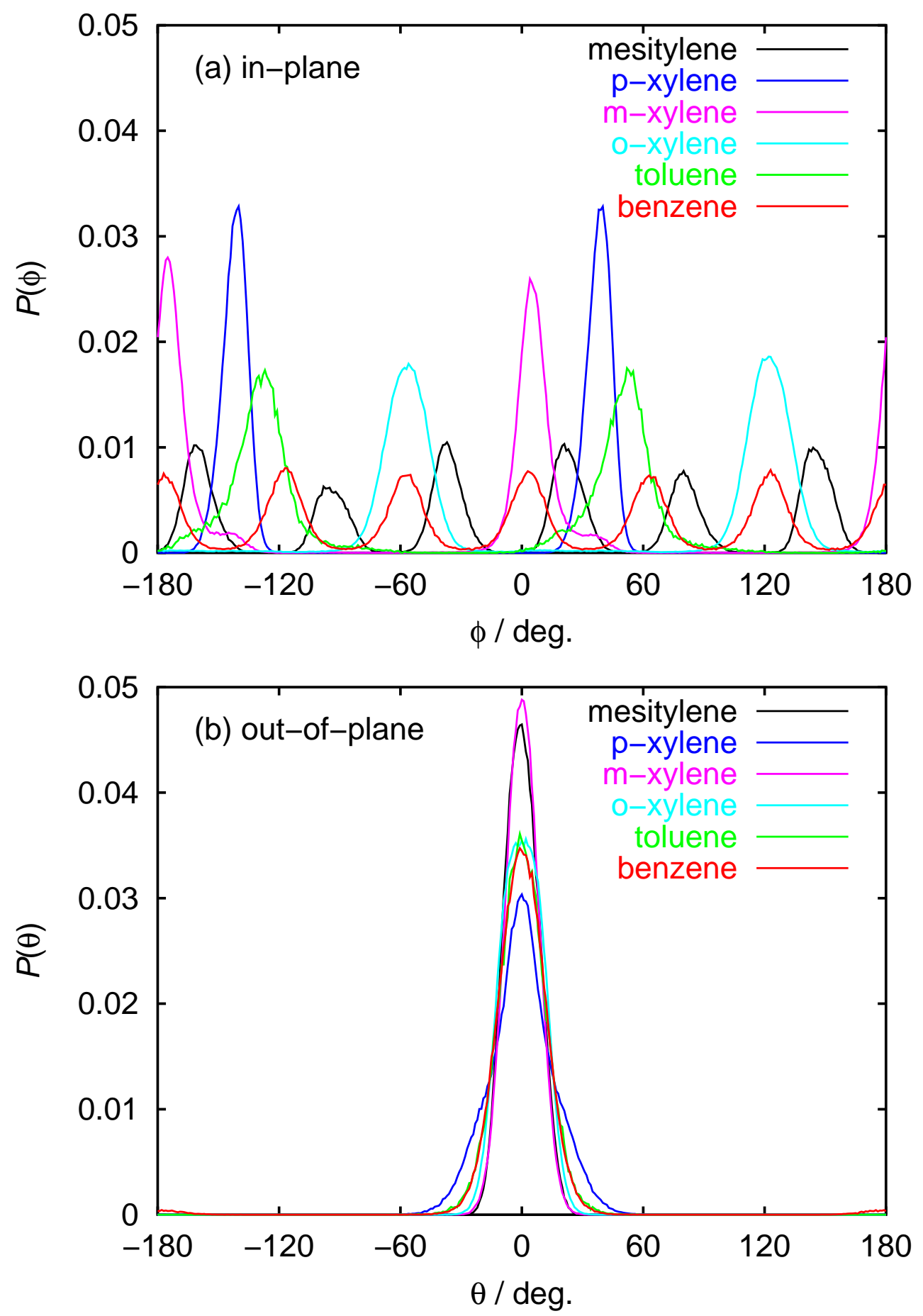
Figure 5 (Y. Tamai et al.)
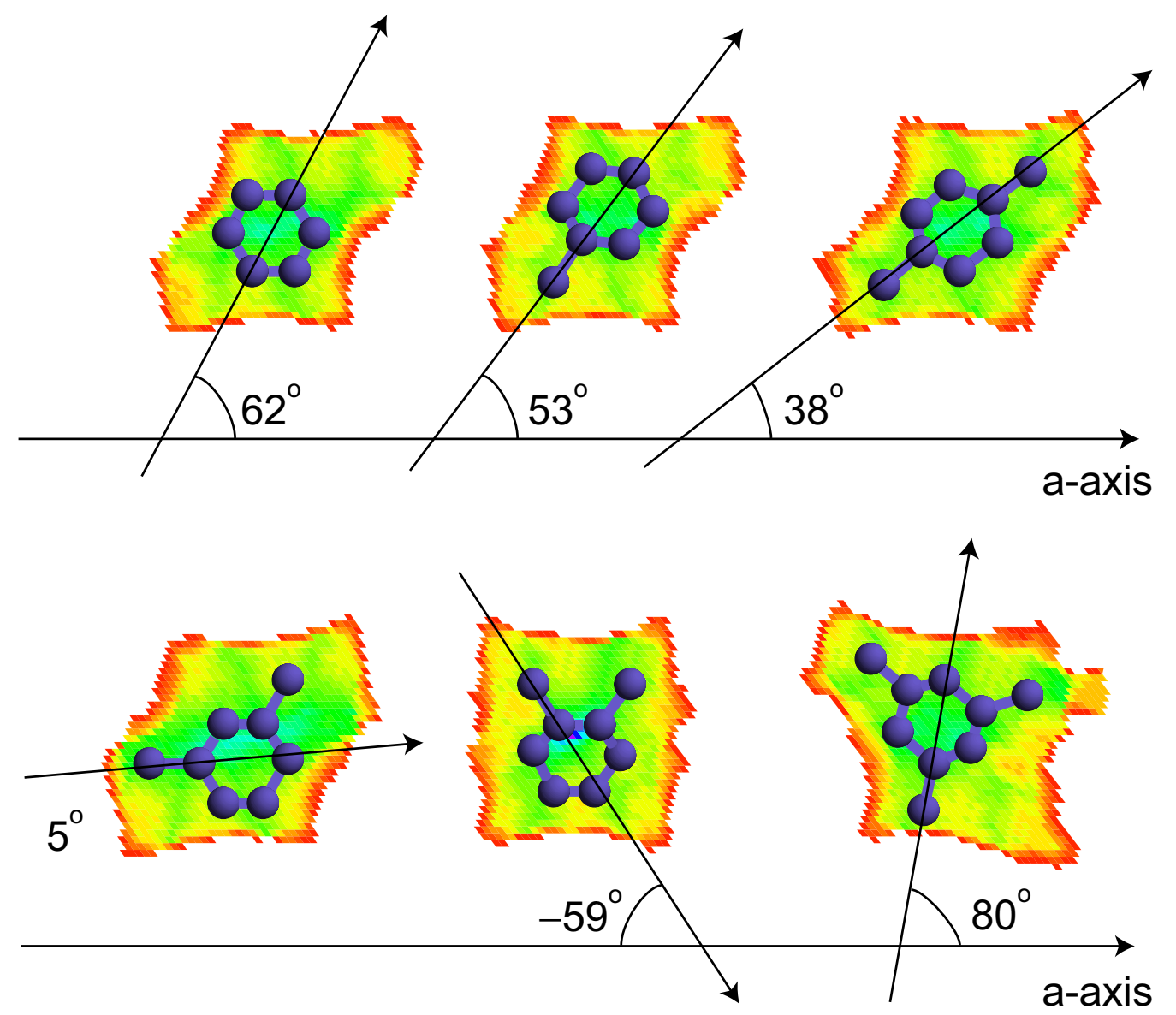
Figure 6 (Y. Tamai et al.)
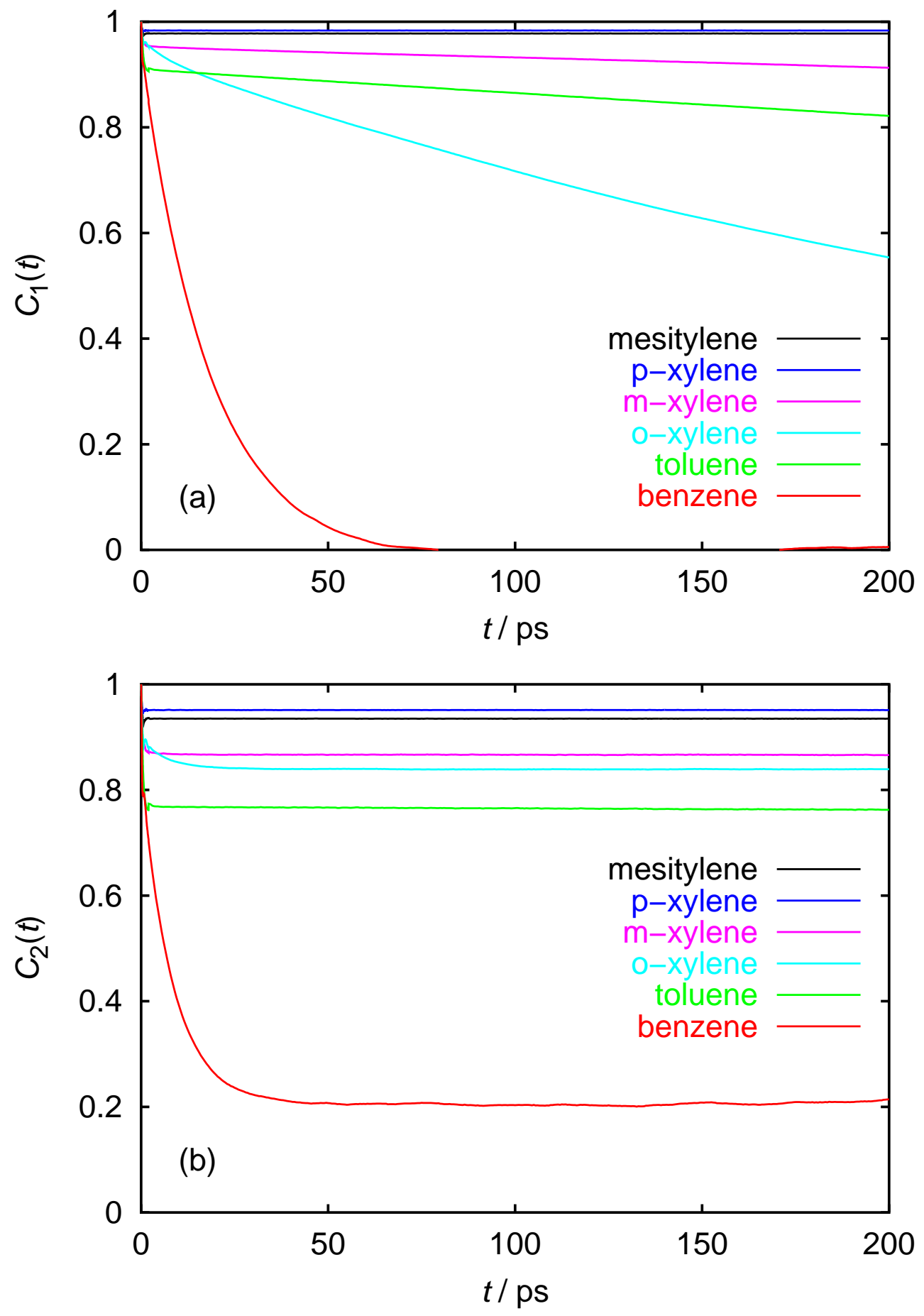
Figure 7 (Y. Tamai et al.)
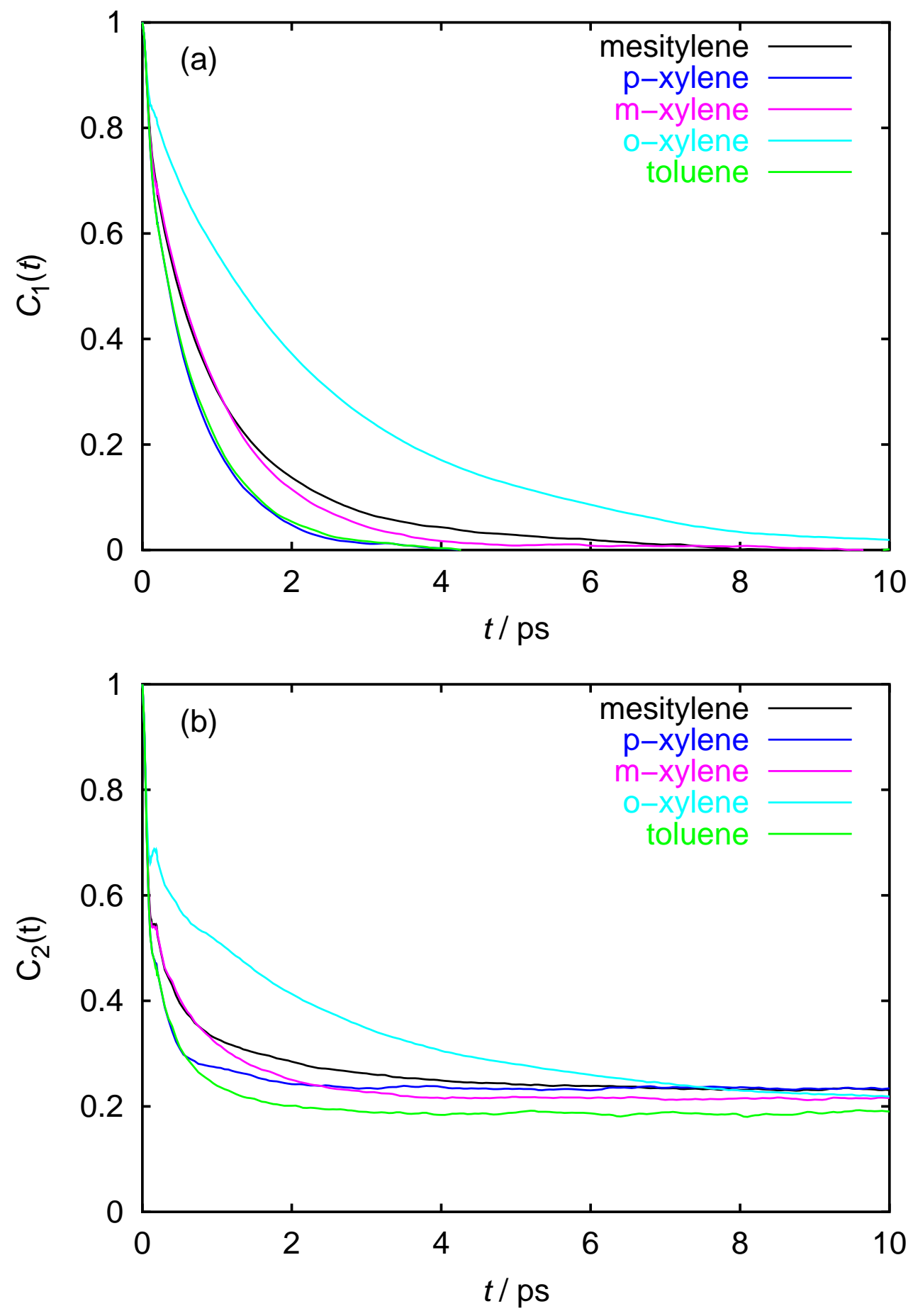
Figure 8 (Y. Tamai et al.)

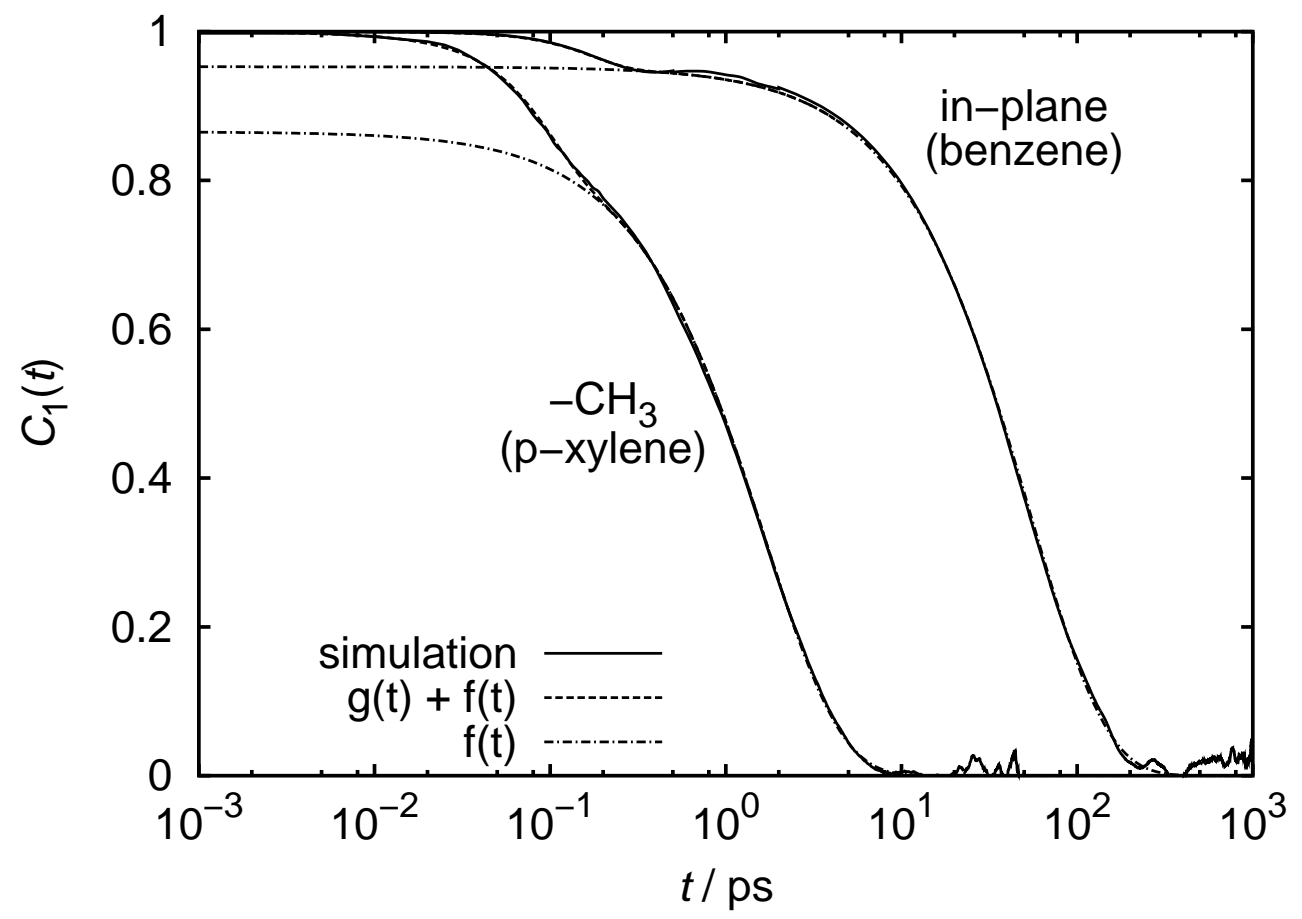


Figure 9 (Y. Tamai et al.)
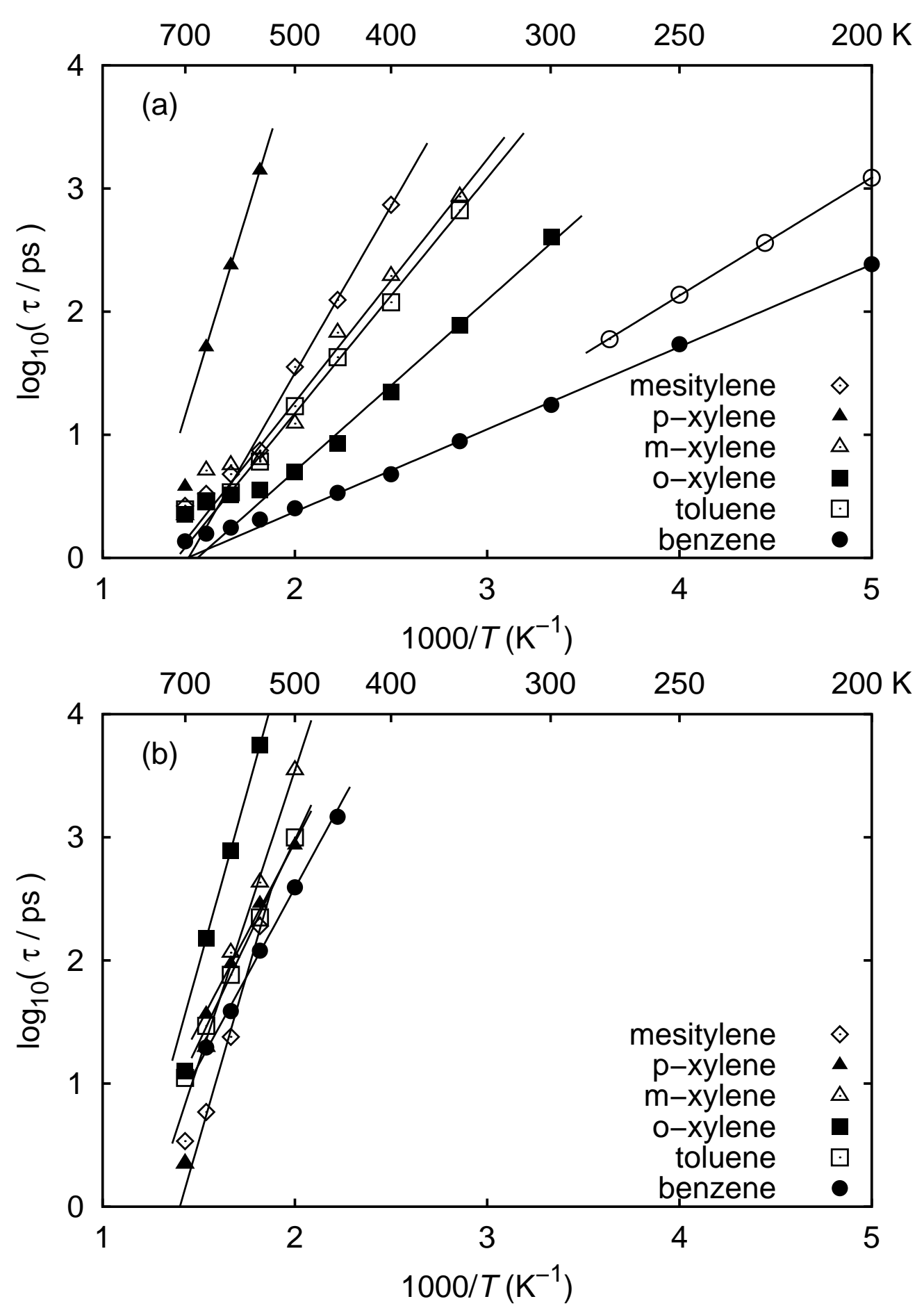
Figure 10 (Y. Tamai et al.)

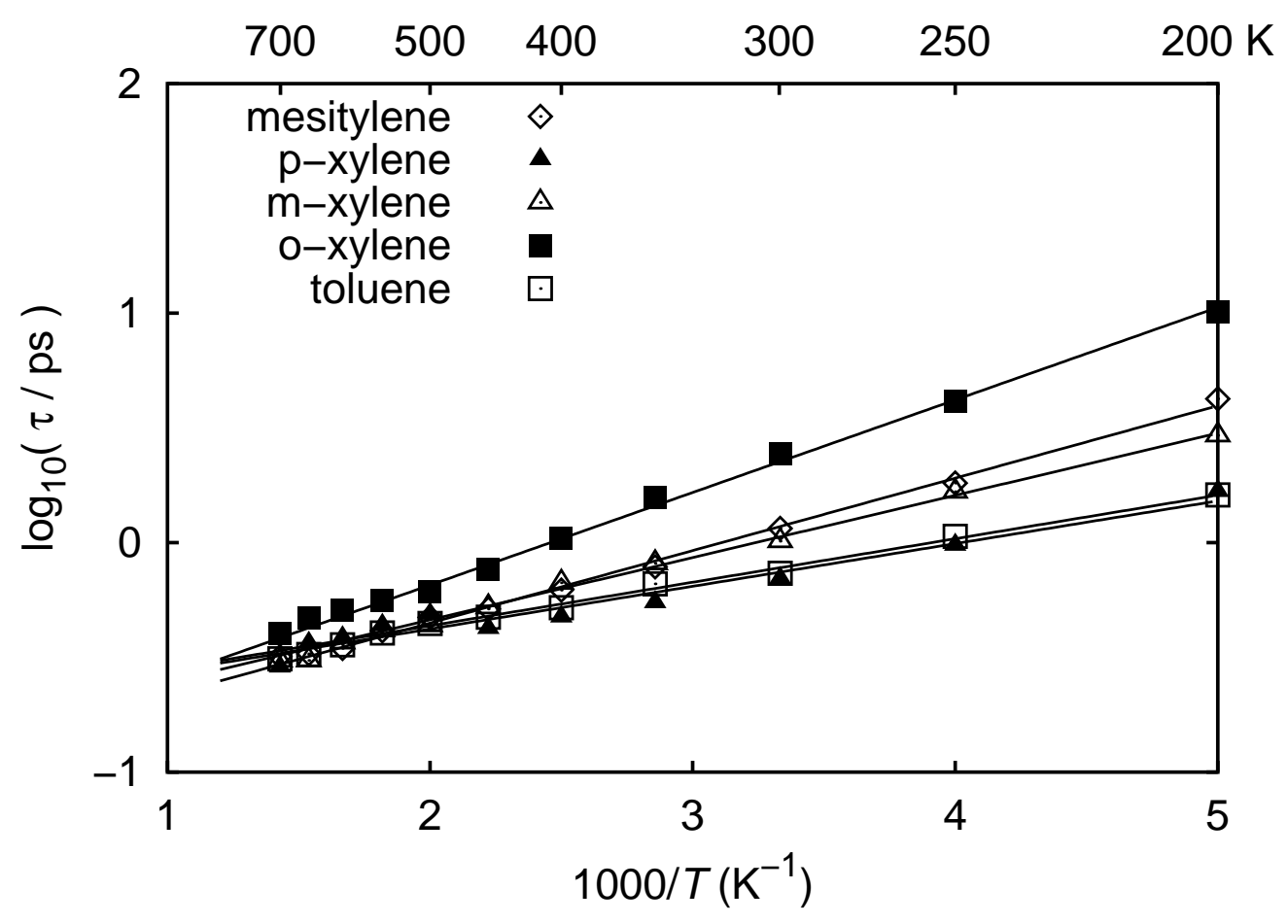

